\title{
A three by three Pascal matrix representations of the generalized Fibonacci and Lucas sequences
}

\author{
Fikri Koken \\ Necmettin Erbakan University, Eregli Kemal Akman Vocational School, Konya, Turkey
}

\begin{abstract}
In this study, a matrix $R_{v}$ is defined, and two closed form expressions of the matrix $R_{v}^{n}$, for an integer $n \geq 1$, are evaluated by the matrix functions in matrix theory. These expressions satisfy a connection between the generalized Fibonacci and Lucas numbers with the Pascal matrices. Thus, two representations of the matrix $R_{v}^{n}$ and various forms of matrix $\left(R_{v}+q \triangle I\right)^{n}$ are studied in terms of the generalized Fibonacci and Lucas numbers and binomial coefficients. By modifying results of $2 \times 2$ matrix representations given in the references of our study, we give various $3 \times 3$ matrix representations of the generalized Fibonacci and Lucas sequences. Many combinatorial identities are derived as applications.
\end{abstract}

Mathematics Subject Classification (2010). 11C20, 11B34, 11B39, 11B65

Keywords. generalized Fibonacci and Lucas sequences, generalized Fibonacci and Lucas matrices, Pascal matrices

\section{Introduction}

The generalized Fibonacci sequence $\left\{U_{n}(p, q)\right\}_{n>0}$ and the generalized Lucas sequence $\left\{V_{n}(p, q)\right\}_{n \geq 0}$, for an integer $n$, are defined by the second order recurrence relation

$$
\begin{aligned}
& U_{n}=p U_{n-1}-q U_{n-2} U_{0}=0, U_{1}=1, \\
& V_{n}=p V_{n-1}-q V_{n-2} V_{0}=2, V_{1}=p,
\end{aligned}
$$

where $p$ and $q\left(p \neq 0\right.$ and $\left.q \neq 0, p^{2} \neq 4 q\right)$ are arbitrary complex coefficients. From the sequences

we derive;

$$
\left\{U_{n}\right\}_{n \geq 0}:=\left\{U_{n}(p, q)\right\}_{n \geq 0} \text { and }\left\{V_{n}\right\}_{n \geq 0}:=\left\{V_{n}(p, q)\right\}_{n \geq 0}
$$

Fibonacci $\left\{F_{n}\right\}_{n>0}:=\left\{U_{n}(1,-1)\right\}_{n>0}$ and Lucas $\left\{L_{n}\right\}_{n \geq 0}:=\left\{V_{n}(1,-1)\right\}_{n \geq 0}$, Pell $\left\{P_{n}\right\}_{n>0}:=\left\{U_{n}(2,-1)\right\}_{n>0}$ and Pell-Lucas $\left\{Q_{n}\right\}_{n>0}:=\left\{V_{n}(2,-1)\right\}_{n>0}$, Jacobsthal $\left\{J_{n}\right\}_{n \geq 0}:=\left\{U_{n}(1,-2)\right\}_{n \geq 0}$ and Jacobsthal Lucas $\left\{j_{n}\right\}_{n \geq 0}:=\left\{V_{n}(2,-1)\right\}_{n \geq 0}$, sequences.

Any $n$-th entries of the sequences $\left\{U_{n}\right\}_{n \geq 0}$ and $\left\{V_{n}\right\}_{n \geq 0}$ are generalized Fibonacci number $U_{n}$ and Lucas number $V_{n}$, respectively, are given by

$$
U_{n}=\left(\alpha^{n}-\beta^{n}\right) /(\alpha-\beta), V_{n}=\alpha^{n}+\beta^{n},
$$

Email addresses: kokenfikri@gmail.com, fkoken@konya.edu.tr (F. Koken)

Received: 09.11.2018; Accepted: 23.12.2019 
where $\alpha=\left(p+\sqrt{p^{2}-4 q}\right) / 2$ and $\beta=\left(p-\sqrt{p^{2}-4 q}\right) / 2$ are the roots of the equation $x^{2}-p x+q=0$. These formulas given in (1.3) are called Binet's formulas [18]. Throughout this paper, we let $n$ be an arbitrary positive integer, $\Delta=p^{2}-4 q, \alpha=(p+\sqrt{\Delta}) / 2$, and $\beta=(p-\sqrt{\Delta}) / 2$, where $p$ and $q\left(p \neq 0\right.$ and $\left.q \neq 0, p^{2} \neq 4 q\right)$ are arbitrary complex coefficients.

Many authors have studied various fundamental properties, matrix representations, and sums of their squares or products of consecutive numbers $U_{n}$ and $V_{n}$ (see, e.g., $[2,4,8,12$, 18, 19]).

Among the generalized Fibonacci and Lucas sequences, the Fibonacci $\left\{F_{n}\right\}_{n>0}$ and Lucas $\left\{L_{n}\right\}_{n \geq 0}$ sequences have achieved a kind of celebrity status, and have been studied extensively in number theory, matrix theory, and applied mathematics (see, e.g., [3, 5, 6, $13,14,17,20,21])$.

In [5] and [6], the authors relate with altering sums of squares of odd and even terms of the Fibonacci sequence and altering sums of their products to the product of the appropriate Fibonacci and Lucas numbers. In [17], the authors give elementary methods to investigate the reciprocal sums of products of two Fibonacci numbers in several ways. Similar formulas for other special sequences such as the Pell, Pell Lucas, Jacobsthal, and Jacobsthal Lucas sequences are obtained by the same methods in $[7,9,10]$.

In [1] and [2], H. Belbachir and F. Bencherif give a number of formulas for sums and alternating sums of product of the generalized Fibonacci and Lucas numbers. These studies extend all results, and recover more easily as the methods in [5-7,9,10]. In [8], Z. Cerin achieves explicit formulas for sums of products of a fixed number of the consecutive generalized Fibonacci and Lucas numbers. These formulas are related to the results given in [2], on the other hand, the author eliminates all restrictions.

An existing formula for any $n$-th power of a $m \times m$ matrix or particular matrices with various matrix identities etc. can also be used to derive various combinatorial identities. In [15], J. Mc Laughlin shows how to derive various combinatorial identities by using a formula for any $n$-th power of a $2 \times 2$ matrix. As an illustration, the well-known $2 \times 2$ Fibonacci matrix $Q^{n}$ gives the ninety-first formula from Vajda's list in [21] and various formulas can be similarly derived by this method. As other formulas for any $n$-th power of matrices of order 2 , we see that many properties of the Fibonacci and Lucas sequences are derived by the Fibonacci matrix $Q^{n}[20]$ and Lucas matrix $Q_{L}^{n}$ [14]. These properties in the context of $2 \times 2$ matrices $U(p, q)$ and $V(p, q)$ associated with the numbers $U_{n}(p, q)$ and $V_{n}(p, q)$ are generalized by using the identities of these numbers in induction method, it can be shown that the matrices $U^{n}(p, q)$ and $V^{n}(p, q)$ are the generalized Fibonacci and Lucas matrices, all elements of which are related to indices of the numbers $U_{n}$ and $V_{n}$ [4]. Also, several properties of the generalized Fibonacci sequence $\left\{H_{k, n}\right\}$ are given by using the same matrix methods [22].

In [19], Melham shows that any $n$-th integer powers of the matrix $R$ of order 3 is related with the numbers $U_{n}(p, q)$, and applies some matrix functions to obtain new infinite sums to the matrix $R^{n}\left(n \in \mathbb{Z}^{+}\right)$, which is derived as

$$
R^{n}=\left(\begin{array}{ccc}
q^{2} U_{n-1}^{2} & q^{2} U_{n-1} U_{n} & q^{2} U_{n}^{2} \\
-2 q U_{n-1} U_{n} & -q\left(U_{n}^{2}+U_{n-1} U_{n+1}\right) & -2 q U_{n} U_{n+1} \\
U_{n}^{2} & U_{n} U_{n+1} & U_{n+1}^{2}
\end{array}\right) .
$$

In [16], the authors obtain a general polynomial identity in $k$ variables, a closed form expression for the entries of the powers of a $k \times k$ matrix is given by using this identity for $k \geq 2$ an arbitrary positive integer. Various combinatorial identities are also derived by using these results.

In [1], H. Belbachir and F. Bencherif derive a formula expressing the general term of a linear recurrent sequence. This result generalizes the result of J. Mc Laughlin about the 
powers of a $2 \times 2$ matrix in [15] to the case of a $m \times m$ matrix, $m \geq 2$. These results are used to derive various identities concerning the Fibonacci and Stirling numbers and combinatorial relations.

In this work we aim to find different relations between matrices containing sequences alike the generalized Fibonacci and Lucas sequences. As far as we know in the literature, matrix representations of the generalized Fibonacci sequence $\left\{U_{n}\right\}_{n>0}$ have been introduced and investigated. We consider matrix representations of the generalized Lucas sequence $\left\{V_{n}\right\}_{n>0}$, which both establish various summation identities involving squares of terms from the sequences $\left\{U_{n}\right\}_{n \geq 0}$ and $\left\{V_{n}\right\}_{n \geq 0}$, and derive properties of the Lucas, Pell Lucas, Jacobsthal Lucas numbers by taking advantage of the ideas introduced for the Fibonacci, Pell, and Jacobsthal numbers in the literature.

\section{The closed form expressions of the matrix $R_{v}^{n}$}

Several generalizations of the Pascal matrix are defined in [23] and a number of theoretic properties associated with the generalized Fibonacci and Lucas sequences and the Pascal-type matrix are studied in [3] and [19]. Unless otherwise stated, $x_{i j}^{n}, i, j=1,2,3$ denotes the entry in the $i$-th row and $j$-th column of $n$-th powers of any matrix $X$ of order 3 , we define first and third column vectors, $\left(u_{i 1}^{n}\right),\left(u_{i 3}^{n}\right),\left(v_{i 1}^{n}\right)$, and $\left(v_{i 3}^{n}\right), i=1,2,3$, including their squares or consecutive product of entries from the sequences $\left\{U_{n}(p, q)\right\}_{n \geq 0}$ and $\left\{V_{n}(p, q)\right\}_{n \geq 0}$, respectively;

$$
\left(u_{i 1}^{n}\right)=\left(\left(\begin{array}{c}
2 \\
i-1
\end{array}\right)\left(-q U_{n-1}\right)^{3-i} U_{n}^{i-1}\right)^{t},\left(u_{i 3}^{n}\right)=\left(\left(\begin{array}{c}
2 \\
i-1
\end{array}\right)\left(-q U_{n}\right)^{3-i} U_{n+1}^{i-1}\right)^{t}, i=1,2,3
$$

and

$$
\left(v_{i 1}^{n}\right)=\left(\left(\begin{array}{c}
2 \\
i-1
\end{array}\right)\left(-q V_{n-1}\right)^{3-i} V_{n}^{i-1}\right)^{t},\left(v_{i 3}^{n}\right)=\left(\left(\begin{array}{c}
2 \\
i-1
\end{array}\right)\left(-q V_{n}\right)^{3-i} V_{n+1}^{i-1}\right)^{t}, i=1,2,3,
$$

where $\left(\begin{array}{l}a \\ b\end{array}\right)$ denotes the binomial coefficient. Now, let us consider a matrix $R_{v}$ of order 3 as

$$
R_{v}=\left(\begin{array}{ccc}
4 q^{2} & 2 p q^{2} & p^{2} q^{2} \\
-4 p q & -q\left(2 p^{2}+\Delta\right) & -2 p q\left(p^{2}-2 q\right) \\
p^{2} & p\left(p^{2}-2 q\right) & \left(p^{2}-2 q\right)^{2}
\end{array}\right), \Delta=p^{2}-4 q
$$

which includes all matrices considered as special cases like Lucas, Pell Lucas, and Jacobsthal Lucas matrices, etc. It is seen that the matrix $R_{v}$ is related with column vectors $\left(u_{i j}^{n}\right)$ and $\left(v_{i j}^{n}\right), j=1,3$ such as

$$
R_{v}\left(u_{i 1}^{n}\right)=\left(v_{i 3}^{n}\right) \text { and } R_{v}\left(v_{i 1}^{n}\right)=\Delta^{2}\left(u_{i 3}^{n}\right), \text { for } i=1,2,3 .
$$

In addition, we present two closed form expressions of $h$-th powers of the matrix $R_{v}$, one of them is the matrix representation.

Theorem 2.1. Let $R_{v}^{h}$ be any $h$-th positive integer powers of matrix given in (2.1). Then,

$$
\begin{aligned}
R_{v}^{2 n} & =\Delta^{2 n}\left(\begin{array}{ccc}
q^{2} U_{2 n-1}^{2} & q^{2} U_{2 n-1} U_{2 n} & q^{2} U_{2 n}^{2} \\
-2 q U_{2 n-1} U_{2 n} & -q\left(U_{2 n}^{2}+U_{2 n-1} U_{2 n+1}\right) & -2 q U_{2 n} U_{2 n+1} \\
U_{2 n}^{2} & U_{2 n} U_{2 n+1} & U_{2 n+1}^{2}
\end{array}\right), \\
R_{v}^{2 n-1} & =\Delta^{2 n-2}\left(\begin{array}{ccc}
q_{2 n-2}^{2} & q^{2} V_{2 n-2} V_{2 n-1} & q^{2} V_{2 n-1}^{2} \\
-2 q V_{2 n-2} V_{2 n-1} & -q\left(V_{2 n-1}^{2}+V_{2 n-2} V_{2 n}\right) & -2 q V_{2 n-1} V_{2 n} \\
V_{2 n-1}^{2} & V_{2 n-1} V_{2 n} & V_{2 n}^{2}
\end{array}\right) .
\end{aligned}
$$

Proof. By using the induction method on $h \in \mathbb{Z}^{+},(2.2)$ and (2.3) are proved according to whether $h$ is even or not.

Firstly, since $R_{v} R_{v}=R_{v}^{2}$ and $R_{v}^{2} R_{v}=R_{v}^{3}$, the matrix $R_{v}^{2}$ for $n=1$ in (2.2) and the matrix $R_{v}^{3}$ for $n=2$ in (2.3) are proved by computing the elements $r_{i 1}^{2}$ and $r_{i 1}^{3}, i=1,2,3$ 
of the matrices $R_{v}^{2}$ and $R_{v}^{3}$, respectively. Other elements can be showed similar to them. The element $r_{11}^{2}$ is $q^{2}\left(p^{2}-4 q\right)^{2}$. Thus, by using $p^{2}-4 q=\Delta$ and $U_{1}=1$, we find that $r_{11}^{2}=\Delta^{2} q^{2}$. The element $r_{21}^{2}$ is $-2 p q\left(-4 q+p^{2}\right)^{2}$. By using $U_{2}=p$, we see that $r_{21}^{2}=-2 \Delta^{2} q U_{2}$. The element $r_{31}^{2}$ is $p^{2}\left(p^{2}-4 q\right)^{2}=\Delta^{2} U_{2}^{2}$. So, the elements $r_{i 1}^{2}, i=1,2,3$ of the matrix $R_{v}^{2}$ are valid for $n=1$. By using $U_{3}=p^{2}-q$, and due to the manner in which elements of the matrix $R_{v}^{2}$ are computed, we note that the matrix $R_{v}^{2}$ is true for $n=1$.

Since $R_{v}^{2} R_{v}=R_{v}^{3}$, the elements $r_{11}^{3}=q^{2} \Delta^{2} V_{2}^{2}, r_{21}^{3}=-2 q \Delta^{2} V_{2} V_{3}, r_{31}^{3}=\Delta^{2} V_{3}^{2}$ are given by using $V_{2}=p^{2}-2 q$ and $V_{3}=p\left(p^{2}-3 q\right)$. Then, the elements $r_{i 1}^{3}, i=1,2,3$ of the matrix $R_{v}^{3}$ are valid for $n=2$. When other elements of the matrix $R_{v}^{3}$ are evaluated by using $V_{4}=p^{4}-4 p^{2} q+2 q^{2}$, we note that the matrix $R_{v}^{3}$ is valid for $n=2$.

Now, we suppose that they are true for $h \leq 2 N, N \geq 2$. Due to $R_{v}^{2 N} R_{v}=R_{v}^{2 N+1}$ or $R_{v}^{2 N-1} R_{v}^{2}=R_{v}^{2 N+1}$, by using the induction hypothesis and $R_{v}$ or $R_{v}^{2}$, we obtain the $R_{v}^{2 N+1}$. Then, elements $r_{i 1}^{2 N+1}, i=1,2,3$ of $R_{v}^{2 N+1}=R_{v}^{2 N} R_{v}$ are given with

$$
\begin{gathered}
r_{11}^{2 N+1}=q^{2}\left(p U_{2 N}-2 q U_{2 N-1}\right)^{2}=q^{2} V_{2 N}^{2}, \\
r_{21}^{2 N+1}=-2 q\left(p U_{2 N}-2 q U_{2 N-1}\right)\left(p U_{2 N+1}-2 q U_{2 N}\right)=-2 q V_{2 N} V_{2 N+1}, \\
r_{31}^{2 N+1}=\left(p U_{2 N+1}-2 q U_{2 N}\right)^{2}=V_{2 N+1}^{2},
\end{gathered}
$$

by using the recurrence relation given in (1.2) and $V_{n}=2 U_{n+1}-p U_{n}=U_{n+1}-q U_{n-1}$. All elements of the $R_{v}^{2 N+1}$ can be proved similar to them.

By using the induction hypothesis and the $R_{v}^{2}$, we can write $R_{v}^{2 N} R_{v}^{2}=R_{v}^{2(N+1)}$. Then, the elements $r_{i 1}^{2 N+2}, i=1,2,3$ of the $R_{v}^{2(N+1)}$ are given by using the recurrence relation given in (1.1), respectively, as

$$
\begin{gathered}
r_{11}^{2 N+2}=q^{2}\left(p U_{2 N}-q U_{2 N-1}\right)^{2}=q^{2} U_{2 N+1}, \\
r_{21}^{2 N+2}=-2 q\left(p U_{2 N+1}-q U_{2 N}\right)\left(p U_{2 N}-q U_{2 N-1}\right)=-2 q U_{2 N+2} U_{2 N+1}, \\
r_{31}^{2 N+2}=\left(p U_{2 N+1}-q U_{2 N}\right)^{2}=U_{2 N+2}^{2} .
\end{gathered}
$$

Other elements can be proved similar to them. Thus, the $R_{v}^{h}$ holds for all positive integer $h$.

Let us mention the properties of the matrix $R_{v}^{h}$;

Remark 2.2. The entries $r_{13}^{2 n}=q^{2} r_{31}^{2 n}$ and $r_{13}^{2 n-1}=q^{2} r_{31}^{2 n-1}$ involve always the numbers $q^{2} U_{2 n}^{2}$ and $q^{2} V_{2 n-1}^{2}$, respectively. And also, the entry $r_{33}^{h}$ involves the numbers $U_{h+1}^{2}$ or $V_{h+1}^{2}$ for even and odd $h$, respectively.

Remark 2.3. The entries $r_{i 1}^{h}, i=1,2,3$ of the matrix $R_{v}^{h}$ are equal to the values of

$$
\Delta^{h}\left(\left(\begin{array}{c}
2 \\
i-1
\end{array}\right)\left(-q U_{h-1}\right)^{3-i} U_{h}^{i-1}\right) \text { and } \Delta^{h-1}\left(\left(\begin{array}{c}
2 \\
i-1
\end{array}\right)\left(-q V_{h-1}\right)^{3-i} V_{h}^{i-1}\right), i=1,2,3
$$

in the expansions $\Delta^{2 n}\left(U_{h}-q U_{h-1}\right)^{2}$ and $\Delta^{2(n-1)}\left(V_{h}-q V_{h-1}\right)^{2}$ for even and odd $h$, respectively. And also, the entries $r_{i 3}^{h}, i=1,2,3$ of $R_{v}^{h}$ are equal to the values of

$$
\Delta^{h}\left(\left(\begin{array}{c}
2 \\
i-1
\end{array}\right)\left(-q U_{h}\right)^{3-i} U_{h+1}^{i-1}\right) \text { and } \Delta^{h-1}\left(\left(\begin{array}{c}
2 \\
i-1
\end{array}\right)\left(-q V_{h}\right)^{3-i} V_{h+1}^{i-1}\right), i=1,2,3
$$


in the expansions $\Delta^{h}\left(U_{h+1}-q U_{h}\right)^{2}$ and $\Delta^{h-1}\left(V_{h+1}-q V_{h}\right)^{2}$ for even and odd $h$, respectively. That is, for $i=1,2,3$,

$$
\begin{aligned}
& r_{i 1}^{h}=\Delta^{h}\left(\left(\begin{array}{c}
2 \\
i-1
\end{array}\right)(-q)^{3-i} U_{h-1}^{3-i} U_{h}^{i-1}\right) \text { for even } h, \\
& r_{i 1}^{h}=\Delta^{h-1}\left(\left(\begin{array}{c}
2 \\
i-1
\end{array}\right)(-q)^{3-i} V_{h-1}^{3-i} V_{h}^{i-1}\right) \text { for odd } h,
\end{aligned}
$$

where $\left(\begin{array}{l}a \\ b\end{array}\right)$ is a positive integer known as a binomial coefficient. The matrix $R_{v}^{h}$ can be expressed as a Pascal-type matrix [23].

The other closed form expression is a polynomial representation, $R_{v}^{h}=c_{0} I+c_{1} R_{v}+c_{2} R_{v}^{2}$, the coefficients $c_{0}, c_{1}$, and $c_{2}$ are given with the numbers $U_{h}$ and $V_{h}$ based on the eigenvalues $\lambda_{j}, j=1,2,3$ of the matrix $R_{v}$. Let us suppose that it is a function $f(x)=x^{n}, n \in \mathbb{Z}^{+}$, then, polynomial expression $f\left(R_{v}\right)$ gives $R_{v}^{n}$ as

$$
f\left(R_{v}\right)=\sum_{i=1}^{3} \prod_{\substack{j=1 \\ i \neq j}}^{3} \frac{f\left(\lambda_{i}\right)}{\lambda_{i}-\lambda_{j}}\left[R_{v}-\lambda_{j} I\right],
$$

where $I$ is the $3 \times 3$ identity matrix. The polynomial $\lambda^{3}-\left(p^{2}-3 q\right) \Delta\left(\lambda^{2}-q \Delta \lambda\right)+q^{3} \Delta^{3}$ is the characteristic polynomial of $R_{v}$, and so, $\lambda_{1}=\Delta \alpha^{2}, \lambda_{2}=-\Delta \alpha \beta, \lambda_{3}=\Delta \beta^{2}$ are the eigenvalues of the matrix $R_{v}$ [11].

Theorem 2.4. Let $R_{v}^{h}$ be any h-th positive integer power of the matrix given in (2.1), and $I$ is the $3 \times 3$ identity matrix. Then,

$$
\begin{array}{r}
R_{v}^{2 n}=\frac{\Delta^{2 n-2}}{p^{2}}\left[U_{2 n} V_{2 n-1} R_{v}^{2}+q \Delta^{2} U_{2 n} U_{2 n-2} R_{v}-q^{3} \Delta^{2} U_{2 n-2} V_{2 n-1} I\right], \\
R_{v}^{2 n-1}=\frac{\Delta^{2 n-3}}{p^{2}}\left[U_{2 n-2} V_{2 n-1} R_{v}^{2}+q \Delta V_{2 n-1} V_{2 n-3} R_{v}-q^{3} \Delta^{2} U_{2 n-2} V_{2 n-3} I\right] .
\end{array}
$$

Proof. By inserting the eigenvalues $\lambda_{1}=\Delta \alpha^{2}, \lambda_{2}=-\Delta q, \lambda_{3}=\Delta \beta^{2}$ into (2.4), we consider it as two equations according to whether $h$ is even or odd, and rewrite the right hand side of $f\left(R_{v}\right)=R_{v}^{h}$ by grouping similar members with respect to matrices $R_{v}^{2}, R_{v}$, and $I$,

$$
\begin{gathered}
R_{v}^{2 n}=\frac{\Delta^{2 n-2}}{p^{2}}\left[\begin{array}{c}
\left(\frac{\alpha^{4 n-1}}{\sqrt{\Delta}}+q^{2 n-1}-\frac{\beta^{4 n-1}}{\sqrt{\Delta}}\right) R_{v}^{2}+\Delta\left(\beta \alpha^{4 n-1}-\left(p^{2}-2 q\right) q^{2 n-1}\right. \\
\left.+\alpha \beta^{4 n-1}\right) R_{v}-q \Delta^{2}\left(\frac{\beta^{2} \alpha^{4 n-1}}{\sqrt{\Delta}}-q^{n}-\frac{\alpha^{2} \beta^{4 n-1}}{\sqrt{\Delta}}\right) I
\end{array}\right], \\
R_{v}^{2 n-1}=\frac{\Delta^{2 n-3}}{p^{2}}\left[\begin{array}{c}
\left(\frac{\alpha^{4 n-3}}{\sqrt{\Delta}}-q^{2 n-2}-\frac{\beta^{4 n-3}}{\sqrt{\Delta}}\right) R_{v}^{2}+\Delta\left(\beta \alpha^{4 n-3}+\left(p^{2}-2 q\right) q^{2 n-2}\right. \\
\left.+\alpha \beta^{4 n-3}\right) R_{v}-q \Delta^{2}\left(\frac{\beta^{2} \alpha^{4 n-3}}{\sqrt{\Delta}}+q^{2 n-1}-\frac{\alpha^{2} \beta^{4 n-3}}{\sqrt{\Delta}}\right) I
\end{array}\right] .
\end{gathered}
$$

We arrange these equations according to the Binet's formulas given in (1.3) and we get the desired results;

$$
\begin{aligned}
& R_{v}^{2 n}=\frac{\Delta^{2 n-2}}{p^{2}}\left[\begin{array}{c}
\frac{\alpha^{2 n}-\beta^{2 n}}{\sqrt{\Delta}}\left(\alpha^{2 n-1}+\beta^{2 n-1}\right) R_{v}^{2}+q \Delta\left(\alpha^{2 n}-\beta^{2 n}\right) \\
\left(\alpha^{2 n-2}-\beta^{2 n-2}\right) R_{v}-q^{3} \Delta^{2} \frac{\alpha^{2 n-2}-\beta^{2 n-2}}{\sqrt{\Delta}}\left(\alpha^{2 n-1}+\beta^{2 n-1}\right) I
\end{array}\right], \\
& R_{v}^{2 n-1}=\frac{\Delta^{2 n-3}}{p^{2}}\left[\begin{array}{c}
\frac{\alpha^{2 n-2}-\beta^{2 n-2}}{\sqrt{\Delta}}\left(\alpha^{2 n-1}+\beta^{2 n-1}\right) R_{v}^{2}+q \Delta\left(\alpha^{2 n-3}+\beta^{2 n-3}\right) \\
\left(\alpha^{2 n}+\beta^{2 n}\right) R_{v}-q^{3} \Delta^{2} \frac{\alpha^{2 n-2}-\beta^{2 n-2}}{\sqrt{\Delta}}\left(\alpha^{2 n-3}+\beta^{2 n-3}\right) I
\end{array}\right] .
\end{aligned}
$$

Remark 2.5. Equating all the entries of the closed form given in (2.2) with (2.5), and (2.3) with (2.6) we obtain several identities of the numbers $U_{n}$ and $V_{n}$. 
Remark 2.6. The matrix equations such that $R_{v}^{n} R_{v}^{m}=R_{v}^{n+m}, R_{v}^{n} R_{v}^{n+1}=R_{v}^{2 n+1}$, and $R_{v}^{n} R_{v}^{-m}=R_{v}^{n-m}$ etc., give some identities of sums or difference of indices for their squares or products of consecutive numbers $U_{n}$ and $V_{n}$. Since these identities and their proofs can be carried out by means of analogous arguments considering by many mathematician, we state only the essential details and omit the identities, and their proofs.

\section{The closed form expressions of the matrix $\left(R_{v}+q \Delta I\right)^{n}$}

Throughout Section 3, let $I$ denote the $3 \times 3$ identity matrix and $n$ be an arbitrary positive integer. From the Cayley Hamilton theorem, $R_{v}^{3}-\left(p^{2}-3 q\right) \Delta\left(R_{v}^{2}-q \Delta R_{v}\right)+$ $q^{3} \Delta^{3} I=0$ is valid, multiplying with $R_{v}^{n}$ of this matrix equation yields

$$
R_{v}^{n+3}+q^{3} \Delta^{3} R_{v}^{n}=\frac{\Delta V_{3}}{p}\left(R_{v}^{n+2}+q \Delta R_{v}^{n+1}\right) .
$$

Remark 3.1. Various identities can be obtained from the cases whether $n$ is even integer or not in (3.1). If $n=2 k, k \geq 1$, then

$$
\begin{aligned}
p V_{n+3}^{2}+q^{3} p \Delta U_{n}^{2} & =V_{3}\left(\Delta U_{n+2}^{2}+q V_{n+1}^{2}\right), \\
q p V_{n+2} V_{n+3}+q^{3} p \Delta U_{n-1} U_{n} & =V_{3}\left(\Delta U_{n+1} U_{n+2}+V_{n} V_{n+1}\right),
\end{aligned}
$$

and, if $n=2 k-1, k \geq 1$, then

$$
\begin{aligned}
p \Delta U_{n+3}^{2}+q^{3} p V_{n}^{2} & =V_{3}\left(V_{n+2}^{2}+q \Delta U_{n+1}^{2}\right), \\
p \Delta U_{n+2} U_{n+3}+q^{3} p V_{n-1} V_{n} & =V_{3}\left(V_{n+1} V_{n+2}+q \Delta U_{n} U_{n+1}\right) .
\end{aligned}
$$

Similar identities can also be given with matrix equation

$$
R_{v}^{n+3}+q^{3} \Delta^{3} R_{v}^{n}=\frac{\Delta V_{3}}{p} R_{v}^{n+1}\left(R_{v}+q \Delta I\right) .
$$

After a little algebraic manipulation on the expansion $\left(R_{v}+q \Delta I\right)^{3}$, we obtain a matrix equation as

$$
\left(R_{v}+q \Delta I\right)^{3}=\Delta p^{2} R_{v}\left(R_{v}+q \Delta I\right) .
$$

Now, we give closed form expressions and some matrix equations on the matrix $\left(R_{v}+q \Delta I\right)^{n}$ to produce summation identities involving terms from the sequences $\left\{U_{n}\right\}$ and $\left\{V_{n}\right\}$.

Theorem 3.2. Let $R_{v}^{n}$ be any $n$-th powers of the matrix given in (2.1), $0 \neq p, q \in \mathbb{C}$, $\Delta=p^{2}-4 q$, and $X:=\left[R_{v}+q \Delta I\right]$ is a matrix $X=\left[x_{i j}\right]_{3 \times 3}$. Then,

$$
\begin{aligned}
X^{2 n+1} & =\Delta^{n} p^{2 n} R_{v}^{n}\left(R_{v}+q \Delta I\right), \\
X^{2 n+2} & =p^{2 n+2} \Delta^{2 n+1}\left[(-q)^{3-i}\left(\begin{array}{c}
2 \\
i-1
\end{array}\right) V_{2 n+i+j-2}\right]_{3 \times 3} .
\end{aligned}
$$

Proof. By using the induction method on $n$ and $\left(R_{v}+q \Delta I\right)^{3}=\Delta p^{2} R_{v}\left(R_{v}+q \Delta I\right)$ given in (3.2), the relation (3.3) can be proved. After the equation given in (3.3) is valid, an equation $\left(R_{v}+q \Delta I\right)^{2 n+2}=\Delta^{n} p^{2 n} R_{v}^{n}\left(R_{v}+q \Delta I\right)^{2}$ is established by multiplying the right hand side of the equation (3.3) with $\left(R_{v}+q \Delta I\right)$. Then, by using the matrix $R_{v}^{n}$ in (2.2) and (2.3), the proof is completed.

Theorem 3.3. Let $X:=\left[R_{v}+q \Delta I\right]$ be a matrix $X=\left[x_{i j}\right]_{3 \times 3}$ and $0 \neq p, q \in \mathbb{C}, \Delta=$ $p^{2}-4 q$, then

$$
X^{n}=p^{n} \Delta^{n-1}\left[(-q)^{3-i}\left(\begin{array}{c}
2 \\
i-1
\end{array}\right) V_{n+i+j-4}\right]_{3 \times 3}, i, j=1,2,3 .
$$


Proof. By using the induction method on $n$ with the following identities

$$
\begin{aligned}
p V_{n+2}-4 q V_{n+1}+p q V_{n} & =\Delta V_{n+1}, \\
V_{n} p^{3}-2 V_{n-1} p^{2} q+V_{n-2} p q^{2}-3 V_{n} p q+4 V_{n-1} q^{2} & =\Delta V_{n+1},
\end{aligned}
$$

the desired results can be proved.

In [2], the authors establish several formulas for sums and alternating sums of products of generalized Fibonacci and Lucas numbers. Especially, the authors extend, more easily, some results of Z. Čerin [5-7,9,10]. By equating the entries $r_{21}^{2 n+1}$ and $r_{31}^{2 n+1}$ of matrices given in (3.3), (3.4), and (3.5), we derive new finite sums involving their squares or products of terms from the generalized Fibonacci and Lucas sequences $\left\{U_{n}\right\}$ and $\left\{V_{n}\right\}$.

Theorem 3.4. If $n=2 k, k \geq 1$, then

$$
\begin{aligned}
\sum_{i=0}^{k}\left(\begin{array}{c}
n \\
2 i
\end{array}\right) q^{2 i} \Delta U_{n-2 i}^{2}+\sum_{i=1}^{k}\left(\begin{array}{c}
n \\
2 i-1
\end{array}\right) q^{2 i-1} V_{n-2 i+1}^{2} & =p^{n} V_{n}, \\
\sum_{i=0}^{k}\left(\begin{array}{c}
n \\
2 i
\end{array}\right) q^{2 i} \Delta U_{n-2 i-1} U_{n-2 i}+\sum_{i=1}^{k}\left(\begin{array}{c}
n \\
2 i-1
\end{array}\right) q^{2 i-1} V_{n-2 i} V_{n-2 i+1} & =p^{n} V_{n-1},
\end{aligned}
$$

and if $n=2 k-1, k \geq 1$, then

$$
\begin{aligned}
\sum_{i=0}^{k}\left(\begin{array}{c}
n \\
2 i
\end{array}\right) q^{2 i} V_{n-2 i}^{2}+\sum_{i=1}^{k}\left(\begin{array}{c}
n \\
2 i-1
\end{array}\right) q^{2 i-1} \Delta U_{n-2 i+1}^{2} & =p^{n} V_{n}, \\
\sum_{i=0}^{k}\left(\begin{array}{c}
n \\
2 i
\end{array}\right) q^{2 i} V_{n-2 i-1} V_{n-2 i}+\sum_{i=1}^{k}\left(\begin{array}{c}
n \\
2 i-1
\end{array}\right) q^{2 i-1} \Delta U_{n-2 i} U_{n-2 i+1} & =p^{n} V_{n-1} .
\end{aligned}
$$

Proof. Let $X:=\left[R_{v}+q \Delta I\right]$ be a matrix $X=\left[x_{i j}\right]_{3 \times 3}$, by using the binomial formula for the left hand side of the expression given in (3.5), we rewrite

$$
X^{n}=\sum_{t=0}^{n}\left(\begin{array}{l}
n \\
t
\end{array}\right)(q \Delta)^{n-t} R_{v}^{t}=p^{n} \Delta^{n-1}\left[(-q)^{3-i}\left(\begin{array}{c}
2 \\
i-1
\end{array}\right) V_{n+i+j-4}\right]_{3 \times 3}, i, j=1,2,3 .
$$

The desired results are obtained by equating the entries $(2,1)$ and $(3,1)$ of the appropriate matrices given in $(2.2)$ or $(2.3)$ on the above results.

By using the similar steps, we establish the following matrix equations.

Corollary 3.5. Let $R_{v}^{n}$ be any $n$-th powers of matrix given in (2.1), and $0 \neq p, q \in \mathbb{C}$, $\Delta=p^{2}-4 q \neq 0$. Then,

$$
p^{2 n} \Delta^{n} R_{v}^{n}\left[R_{v}+q \Delta I\right]^{n}=\left[R_{v}+q \Delta I\right]^{3 n} .
$$

Proof. By using the techniques given above and the well-known identities $V_{2 n-1} U_{2 n+1}-$ $q V_{2 n-2} U_{2 n}=V_{4 n-1}, V_{2 n} U_{2 n+1}-q V_{2 n-1} U_{2 n}=V_{4 n}$ and $V_{2 n-2} V_{2 n}-q V_{2 n-1} V_{2 n-3}=\Delta U_{4 n-3}$, the desired result is obtained.

We also observe that the following identity is valid;

$$
\left[R_{v}^{3}+q^{3} \Delta^{3} I\right]^{n}=\frac{\Delta^{n} V_{3}^{n}}{p^{n}} R_{v}^{n}\left[R_{v}+q \Delta I\right]^{n} .
$$

Remark 3.6. Manipulating the equation given in (3.6) yields

$$
\left[R_{v}^{3}+q^{3} \Delta^{3} I\right]^{n}=\frac{\Delta^{n} V_{3}^{n}}{p^{n}} R_{v}^{n}\left[R_{v}+q \Delta I\right]^{n}=\frac{V_{3}^{n}}{p^{3 n}}\left[R_{v}+q \Delta I\right]^{3 n}
$$


and, expanding (3.8) and (3.5) gives

$$
\begin{aligned}
\sum_{i=0}^{n}\left(\begin{array}{c}
n \\
i
\end{array}\right)(q \Delta)^{3(n-i)} R_{v}^{3 i} & =\frac{\Delta^{n} V_{3}^{n}}{p^{n}} \sum_{i=0}^{n}\left(\begin{array}{c}
n \\
i
\end{array}\right)(q \Delta)^{(n-i)} R_{v}^{n+i} \\
& =\Delta^{3 n-1} V_{3}^{n}\left(\begin{array}{ccc}
q^{2} V_{3 n-2} & q^{2} V_{3 n-1} & q^{2} V_{3 n} \\
-2 q V_{3 n-1} & -2 q V_{3 n} & -2 q V_{3 n+1} \\
V_{3 n} & V_{3 n+1} & V_{3 n+2}
\end{array}\right)
\end{aligned}
$$

Many identities similar to the identities given below can be established by using the matrix equation given in (3.10).

Theorem 3.7. The following identities are valid:

$$
\begin{aligned}
& \sum_{i=0}^{k}\left[\left(\begin{array}{c}
n \\
2 i
\end{array}\right) q^{n-2 i} \Delta U_{n+2 i}^{2}+\left(\begin{array}{c}
n \\
2 i+1
\end{array}\right) q^{n-2 i-1} V_{n+2 i+1}^{2}\right]=p^{n} V_{3 n}, n=2 k, \\
& \sum_{i=0}^{k-1}\left[\left(\begin{array}{c}
n \\
2 i
\end{array}\right) q^{n-2 i} V_{n+2 i}^{2}+\left(\begin{array}{c}
n \\
2 i+1
\end{array}\right) q^{n-2 i-1} \Delta U_{n+2 i+1}^{2}\right]=p^{n} V_{3 n}, n=2 k-1 .
\end{aligned}
$$

Proof. By equating the $(3,1)$ entry of the matrix given in $(3.10)$ with the $(3,1)$ entry of (3.9) which is obtained by the help of (2.2) if $n$ is even (or (2.3) if $n$ is odd), we obtain the desired results.

\section{Conclusion}

In this paper, two different closed forms of the matrix functions $f\left(R_{v}\right)=R_{v}^{n}$ are introduced for the generalized Fibonacci and Lucas sequences. Several new identities are obtained for the generalized Fibonacci and Lucas numbers from these closed forms. The generalized Lucas matrix is also described by the matrix $\left(R_{v}+q \Delta I\right)^{n}$. From odd and even cases of $n$, many combinatorial identities are obtained.

\section{References}

[1] H. Belbachir and F. Bencherif, Linear recurrent sequences and powers of a square matrix, Integers, 6 (A12), 2006.

[2] H. Belbachir and F. Bencherif, Sums of product of generalized Fibonacci and Lucas numbers, Ars Combin. 110, 33-43, 2013.

[3] H. Belbachir and L. Szalay, Fibonacci and Lucas Pascal triangles, Hacet. J. Math. Stat. 45 (5), 1343-1354, 2016.

[4] G. Cerda-Morales, On generalized Fibonacci and Lucas numbers by matrix methods, Hacet. J. Math. Stat. 42 (2), 173-179, 2013.

[5] Z. Čerin, Alternating sums of Fibonacci products, Atti Semin. Mat. Fis. Univ. Modena Reggio Emilia, 53 (2), 331-344, 2005.

[6] Z. Črin, Properties of odd and even terms of the Fibonacci sequence, Demons. Math. 39 (1), 55-60, 2006.

[7] Z. Čerin, Sums of squares and products of Jacobsthal numbers, J. Integer Seq. 10 (7), Art. 2.5, 1-15, 2007.

[8] Z. Čerin, Sums of products of generalized Fibonacci and Lucas numbers, Demons. Math. 42 (2), 247-258, 2009.

[9] Z. Čerin and G.M. Gianella, On sums of squares of Pell-Lucas numbers, Integers, 6 (A15), 1-16, 2006.

[10] Z. Čerin and G.M. Gianella, On sums of Pell numbers, Acc. Sc. Torino-Atti Sc. Fis. 141, 23-31, 2007.

[11] N.J. Higham, Functions of matrices. Theory and computation, Society for Industrial and Applied Mathematics, Philadelphia, 2008. 
[12] N. Irmak and M. Alp, Some identities for generalized Fibonacci and Lucas sequences, Hacet. J. Math. Stat. 42 (4), 331-338, 2013.

[13] F. Koken, The Representations of the Fibonacci and Lucas matrices, Iranian J. Sci. Tech., Trans. A: Sci. 43 2443-2448, 2019.

[14] F. Koken and D. Bozkurt, On Lucas numbers by the matrix method, Hacet. J. Math. Stat. 39 (4), 471-475, 2010.

[15] J. Mc Laughlin, Combinatorial identities deriving from the n-th power of $2 x 2$ matrix, Integers, A19 (4), 1-15, 2004.

[16] J. Mc Laughlin and B. Sury, Powers of matrix and combinatorial identities, Integers A13 (5), 1-9, 2005.

[17] R. Liu and A.YZ. Wang, Sums of products of two reciprocal Fibonacci numbers, Adv. Difference Equ. 136, 1-26, 2016.

[18] E. Lucas, Théorie des fonctions numériques simplement périodiques, Amer. J. Math. 1, 184-240, 1878.

[19] R.S. Melham, Lucas sequences and functions of a 3-by-3 matrix, Fibonacci Quart. 37 (2), 111-116, 1999.

[20] J.R. Silvester, Fibonacci properties by matrix methods, Math. Gaz. 63 (425), 188-191, 1979.

[21] S. Vajda, Fibonacci, Lucas Numbers, and the Golden Section. Theory and Applications. Ellis Horwood Ltd., Chichester; Halsted Press, New York, 1989.

[22] A.A. Wani, G.P.S. Rathore, V.H. Badshah and K. Sisodiya, Two-by-two matrix representation of a generalized Fibonacci sequence, Hacet. J. Math. Stat. 47 (3), 637-648, 2018.

[23] Z. Zhang, The linear algebra of the generalized Pascal matrix, Linear Algebra Appl. 250, 51-60, 1997. 